\title{
Habilidades Sociais e Problemas de Comportamento de Crianças sob Acolhimento Institucional
}

\author{
Livia Lira de Lima Guerra ${ }^{1}$ \\ Zilda Aparecida Pereira Del Prette ${ }^{1}$ \\ ${ }^{1}$ Universidade Federal de São Carlos
}

\begin{abstract}
Resumo
Este estudo realizou uma caracterização do repertório de habilidades sociais e problemas de comportamento de crianças em situação de acolhimento institucional, que foi comparada à média normativa brasileira. Participaram 36 crianças, ambos os gêneros, na faixa etária de 6 a 12 anos e 19 cuidadores, como avaliadores do repertório das crianças, utilizando-se o Inventário de Habilidades Sociais, Problemas de Comportamento e Competência Acadêmica para Crianças (SSRS). Os resultados mostraram que: (a) a amostra de crianças apresentou escores significativamente abaixo da norma em habilidades sociais, com mais da metade delas classificada com repertório deficitário e médio inferior em habilidades sociais, tanto na autoavaliação quanto na avaliação do cuidador; (b) a amostra apresentou repertório altamente comprometido em problemas de comportamento, sendo mais frequentes os do tipo externalizantes. Discute-se a importância e urgência de intervenções voltadas para a promoção de habilidades sociais das crianças institucionalizadas, de modo a prevenir e superar problemas em seu desenvolvimento.

Palavras-chave: crianças, acolhimento institucional, habilidades sociais, problemas de comportamento
\end{abstract}

\section{Social Skills and Behavior Problems of Children under Institutional Care}

\begin{abstract}
This study conducted a characterization of the repertoire of social skills and behavioral problems of children in institutional care, which was compared to the Brazilian normative average. Participants included 36 children, of both genders, from 6 to 12 years old, and 19 caregivers as evaluators of the children's repertoire, using the Social Skills Rating System (SSRS). The results showed that: (a) the sample of children presented scores significantly below the Brazilian average in social skills, with more than half of them classified as having deficient repertoire and lower means in social skills, both in self-assessment and in the caregiver's evaluation; (b) the sample presented a highly compromised repertoire in behavioral problems, and the externalizing type was the most common. It discusses the importance and urgency of interventions aimed at promoting the social skills of institutionalized children, in order to prevent and overcome problems in their development.

Keywords: children; social skills; behavior problems; institutional care
\end{abstract}

Habilidades Sociales e Problemas de Comportamiento de Niños en Acogimiento Institucional

\begin{abstract}
Resumen
Este estudio realizó una caracterización del repertorio de habilidades sociales y problemas de comportamiento de niños en situación de acogimiento institucional, que se comparó con los medios normativos brasileños. Participaron 36 niños de ambos sexos, con edades de 6 a 12 años y 19 cuidadores, como evaluadores del repertorio de los niños, utilizándose el Inventario de Habilidades Sociales, Problemas de Comportamiento y Competencia Académica para Niños SSRS. Los resultados mostraron que: (a) la muestra de niños presentó resultados significativamente por debajo de la norma en habilidades sociales, con más de la mitad de ellas clasificada con repertorio deficitario y medio inferior en habilidades sociales, tanto en la autoevaluación como en la evaluación del cuidador; (b) la muestra presentó un repertorio altamente comprometido en problemas de comportamiento, siendo más frecuentes los del tipo de externalización. Se discute la importancia y urgencia de intervenciones dirigidas a la promoción de habilidades sociales de los niños acogidos en instituciones, para prevenir y superar problemas en su desarrollo. Palabras clave: niños; acogimiento institucional; habilidades sociales; problemas de comportamiento
\end{abstract}

\section{Introdução}

$\mathrm{O}$ acolhimento institucional configura-se como uma medida de proteção preconizada pelo Estatuto da Criança e do Adolescente (ECA, 1990), indicada para crianças e adolescentes cujos direitos básicos foram violados. Tal violação caracteriza-se pela exposição à violência física ou sexual, abandono, pobreza material, vivência de rua, dependência química dos pais, orfandade, dentre outros motivos. Os órgãos de
Justiça, portanto, determinam a suspensão temporária do poder familiar e concedem a guarda do menor ao responsável direto nas instituições de acolhimento. No entanto, além da exposição a tais fatores de risco relacionados ao contexto familiar anterior ao acolhimento, a literatura aponta que o ambiente institucional geralmente não oferece condições propícias para o pleno desenvolvimento de crianças e adolescentes (Bakermans-Kranenburg, Van IJzendoorn, \& Juffer, 2008; Chaves, Lima, Mendonça, Custódio, \& Matias, 2013). 
No estudo de Levin, Fox, Zeanah Jr., e Nelson (2015), os autores encontraram que crianças institucionalizadas apresentaram significativamente maiores dificuldades de comunicação social e padrões de comportamento de "quase-autismo", quando comparadas a crianças nunca institucionalizadas. Os resultados foram reportados pelos cuidadores por meio do Social Communication Questionnaire (SCQ). No entanto, um terceiro grupo, o da intervenção precoce em acolhimento familiar, apresentou melhores habilidades de comunicação social do que aquelas de instituições tradicionais, mas ainda menores que o grupo de crianças nunca institucionalizada.

Outros achados confirmam e expandem as evidências sobre os déficits cognitivos e desenvolvimentais de crianças institucionalizadas, como menor volume do hipocampo (Hodel et al., 2015) e dificuldades na atenção e regulação emocional (Troller-Renfree, McDermott, Nelson, Zeanah, \& Fox, 2015). Adicionalmente, em estudo de metanálise (Lionettia, Pastore, \& Barone, 2015) sobre padrões de apego de 399 crianças institucionalizadas, foram encontrados resultados preocupantes: apenas $8 \%$ de apego seguro contra $28 \%$ de inseguro e $54 \%$ de desorganizado (ou não classificado). Os autores concluíram que, se comparadas com crianças nunca institucionalizadas, as do estudo apresentaram significativamente maior dano no desenvolvimento socioemocional. Conforme Lima e Lima (2012), a carência de estimulação física e social por parte dos ambientes institucionais pode acarretar, ainda, problemas de crescimento e déficit em habilidades mínimas requeridas para a aquisição de novos comportamentos.

Associada a essa carência de estimulação, Bakermans-Kranenburg, Van IJzendoorn e Juffer (2008) destacam também: o elevado número de crianças por abrigo e por cuidador, a alta rotatividade destes e a menor qualidade no cuidado e no estabelecimento de vínculos afetivos. No entanto, embora não se possa isolar, tão facilmente, as causas do atraso no desenvolvimento das crianças institucionalizadas, a privação de experiências socioemocionais positivas tem sido também referida (Cardeira, Almeida, Martins, Bento, \& Cabeleira, 2011).

O desenvolvimento socioemocional inclui vários aspectos a serem considerados. Um deles é a aquisição e aperfeiçoamento das habilidades sociais e competência social, juntamente com cognições, valores, conhecimento sobre o ambiente social e sobre si mesmo, que caracterizam a competência social (Del
Prette \& Del Prette, 2017). A partir do nascimento, inicia-se o desenvolvimento social do indivíduo e há evidências de que, sob condições favoráveis, ou pelo menos não desfavoráveis, seu repertório de habilidades sociais se torna mais elaborado ao longo da vida por meio das constantes interações com o ambiente (Del Prette \& Del Prette, 2011).

A reconhecida influência dos fatores ambientais no desenvolvimento das habilidades sociais é aliada a estudos específicos sobre a predisposição genética para algumas delas, como a empatia (Gaspar, 2014; Justo, Carvalho, \& Kristensen, 2014). Mesmo se reconhecendo uma tendência filogenética para a empatia, o seu desenvolvimento está associado à interação entre diversas variáveis, tais como o temperamento, o desenvolvimento cognitivo, os estilos parentais e o relacionamento entre pais e filhos (Justo et al., 2014). Por isso, desde as primeiras experiências, se as condições ambientais não são favoráveis, consequentemente, podem ocorrer os déficits nesse repertório da criança, comprometendo este e outros aspectos do desenvolvimento socioemocional. As habilidades sociais são apontadas como fator de proteção no curso do desenvolvimento e de qualidade de vida, inclusive para crianças em situação de vulnerabilidade (Cecconello \& Koller, 2000; Del Prette, Del Prette, Gresham, \& Vance, 2012). Assim, diante dos fatores de risco a que estão expostas as crianças em situação de acolhimento institucional, conforme referido antes, justifica-se o investimento em avaliar seu repertório de habilidades sociais, como forma de melhor caracterizar a necessidade de programas para superar déficits nessa área e reduzir a probabilidade de problemas de comportamento e de aprendizagem, geralmente a eles associados.

A hipótese do estudo foi que as crianças institucionalizadas poderiam apresentar um repertório de habilidades sociais abaixo da média normativa brasileira, comprometendo seu desenvolvimento. Assim, o presente artigo teve como objetivos: (1) caracterizar o repertório de habilidades sociais e problemas de comportamento de crianças em situação de acolhimento institucional e (2) analisar possíveis associações entre o repertório de habilidades sociais e problemas de comportamento nessa população específica.

\section{Método}

O presente estudo orientou-se pelos critérios estabelecidos pelo Conselho Nacional de Saúde, no que se 
refere às diretrizes e normas regulamentadoras de pesquisas envolvendo seres humanos (Resolução 466/12), e foi aprovado pelo Comitê de Ética em Pesquisa em Seres Humanos da Universidade Federal de São Carlos (Protocolo 1.474.260). Após a autorização de cada instituição para efetuar a pesquisa e para a participação das crianças, foi solicitado ainda a cada criança a assinatura do Termo de Assentimento Livre e Esclarecido (TALE) e aos cuidadores, o Termo de Termo de Consentimento Livre e Esclarecido (TCLE).

\section{Participantes}

Participaram deste estudo 36 crianças (52,8\% meninos e $47,2 \%$ meninas), na faixa etária dos 6 aos 12 anos e que cursavam do $1^{\circ}$ ao $6^{\circ}$ ano do ensino fundamental (todos em escolas públicas). Participaram ainda 19 cuidadores, entre 23 e 62 anos de idade, com escolaridade variando do ensino fundamental completo até o ensino superior completo, como informantes em relação às crianças. Para as crianças, $O$ critério de inclusão foi o tempo de acolhimento superior a um mês; e o critério de exclusão foi a presença de transtorno grave do desenvolvimento (não houve nenhum caso informado pelos diretores). Para os cuidadores, o critério de inclusão foi o tempo de trabalho na instituição superior a um mês, supondo que esse é um tempo mínimo suficiente para conhecerem as crianças. A idade média das crianças, no momento em que começaram a participar da pesquisa, foi de 9,08 anos $(d p=1,88)$ e, a amostra apresentou uma distribuição equilibrada entre as faixas etária de $6,7,8$ e 9 anos $(n=18 ; 50 \%)$ e 10,11 e 12 anos $(n=18 ; 50 \%)$. Os anos escolares predominantes foram o $4^{\circ}, 5^{\circ}$ e $6^{\circ}$ $(n=23 ; 63,9 \%)$. O motivo do acolhimento mais frequente foi a dependência química dos pais, seguido da negligência e violência doméstica. $O$ tempo médio de institucionalização foi de 15, 69 meses $(d p=19,17)$. Esta pesquisa foi realizada com participantes de quatro instituições de acolhimento, mantidas com verbas municipais e filantrópicas, localizadas em três cidades de médio porte do interior de São Paulo. As coletas ocorreram nas salas utilizadas como bibliotecas, das respectivas instituições. Todas elas são configuradas como abrigo de pequeno porte de atendimento integral, uma delas possui também quatro unidades da modalidade Casa-Lar.

\section{Instrumentos}

Inventário de Habilidades Sociais, Problemas de Comportamento e Competência Acadêmica para Crianças - SSRS
(Gresham \& Elliot, 2016). Trata-se de adaptação de instrumento desenvolvido nos Estados Unidos (Social Skills Rating System), com escalas tipo Likert de três pontos para frequência, variando de 0 (nunca), 1 (algumas vezes) e 2 (muito frequente). $A$ análise psicométrica produziu indicadores de validade e precisão satisfatórios (Freitas \& Del Prette, 2015), além de escores para as diferentes subescalas. No presente estudo, foram utilizadas as versões de autoavaliação da criança e a versão de pais (habilidades sociais e problemas de comportamento) que foi preenchida pelos cuidadores, no papel de informantes a respeito das crianças participantes do estudo. Para isso, foram feitas algumas adaptações de conteúdo dos itens do instrumento original, respeitando-se, no entanto, as classes de habilidades sociais e problemas de comportamento. Substituindo por exemplo: "Usa o tempo livre em casa de maneira aceitável", "Em casa, fala em tom de voz apropriado" e "Guarda seus brinquedos ou outras coisas da casa" por "Usa o tempo livre na instituição de maneira aceitável", "Na instituição, fala em tom de voz apropriado" e "Guarda seus brinquedos ou outras coisas da instituição", respectivamente. Ao longo desse texto será utilizado o termo SSRS-cuidador para se referir a essa versão. Os resultados do alfa de Cronbach, aferidos a partir da amostra do presente estudo foram: 0.69 na escala de autoavaliação da criança e 0,72 e 0,75 nas escalas de habilidades sociais e problemas de comportamento, respectivamente, conforme avaliadas pelo cuidador.

\section{Procedimento}

A coleta de dados com as crianças foi realizada individualmente, de acordo com horários estabelecidos pelas instituições. No início da aplicação, foi realizado um breve rapport, com o intuito de gerar um ambiente favorável, explicar os objetivos do estudo (enfatizando que não havia respostas certas ou erradas) e dar as instruções normativas previstas no manual do instrumento. Os itens do instrumento foram previamente gravados em áudio e, no momento da aplicação, reproduzidos para cada criança por meio de um celular. A pesquisadora anotava as respostas dadas oralmente pelas crianças. O tempo médio de coleta foi de 25 minutos com cada criança. A coleta de dados com os cuidadores também foi individual, de acordo com os horários das instituições. Em todas elas, exceto uma, os cuidadores de referência avaliaram as crianças sob sua responsabilidade. Nessa exceção, o cuidador atribuído para avaliar a criança foi determinado pela coordenação, considerando 
aquele profissional que tinha mais conhecimento sobre a criança.

\section{Tratamento de Dados}

Os dados foram organizados em planilhas do software estatístico Statistical Package for Social Sciences (SPSS), versão 20.0 e convertidos em escores com base nas orientações do manual. As distribuições das variáveis contínuas foram examinadas com medidas descritivas (simetria, curtose, média, desvio padrão, mediana, valores mínimos e máximos), representadas em histogramas e submetidas ao teste de Kolmogorov-Smirnov ${ }^{1}$ para verificar a sua normalidade. De acordo com tais critérios, assumiu-se que os dados foram provenientes de uma população normal e, portanto, poderiam ser analisados com testes paramétricos (Dancey \& Reidy, 2013). Em seguida, procedeu-se às análises descritivas e inferenciais, adotando-se um nível de significância de $5 \%(p \leq 0,05)$. Também foram efetuadas análises de consistência interna das escalas utilizadas, por meio do alfa de Cronbach: 0,69 na escala de autoavaliação da criança e 0,72 e 0,75 nas escalas de habilidades sociais e problemas de comportamento, respectivamente conforme avaliadas pelo cuidador.
Os dados normativos do SSRS foram utilizados para realizar a análise descritiva das habilidades sociais e problemas de comportamento das crianças. De acordo com o escore geral e os escores fatoriais obtidos por cada criança, foi definida a posição percentil correspondente e sua classificação em relação aos dados normativos: repertório deficitário (percentil $\leq 25$ ); médio inferior (percentil $>25$ e $\leq 50$ ); médio superior (percentil $>50$ e $<75$ ); repertório elaborado (percentil $\geq 75$ ). Posteriormente, foram determinadas as frequências absolutas e relativas de crianças que se localizaram nos repertórios considerados como deficitário, médio inferior, médio superior e elaborado para cada categoria da variável correspondente. Para analisar possíveis associações entre o repertório de Habilidades Sociais (HS) e Problemas de Comportamento (PC) das crianças, foi planejada uma matriz de correlações executada por meio do software estatístico SPSS.

\section{Resultados}

Este estudo originou dois tipos de resultados: os descritivos, referentes à caracterização da amostra, e os correlacionais, referentes à relação entre habilidades

Tabela 1.

Dados Descritivos de Habilidades Sociais das Crianças em Relação a Padrões Normativos do SSRS

\begin{tabular}{|c|c|c|c|c|c|}
\hline \multirow{3}{*}{ Habilidade Sociais (HS) } & \multirow{3}{*}{$\begin{array}{l}\text { Variação } \\
\text { possível do } \\
\text { escore }\end{array}$} & \multicolumn{4}{|c|}{ Repertório } \\
\hline & & Deficitário & $\begin{array}{l}\text { Médio } \\
\text { Inferior }\end{array}$ & $\begin{array}{c}\text { Médio } \\
\text { Superior }\end{array}$ & Elaborado \\
\hline & & $n(\%)$ & $n(\%)$ & $n(\%)$ & $n(\%)$ \\
\hline \multicolumn{6}{|l|}{ SSRS-criança } \\
\hline Global de HS & $0-40$ & $18(50)$ & $11(30.6)$ & $4(11.1)$ & $3(8.3)$ \\
\hline F1 Empatia-Afetividade & $0-10$ & $20(55.5)$ & $8(22.2)$ & $6(16.7)$ & $2(5.6)$ \\
\hline F2 Responsabilidade & $0-10$ & $15(41.6)$ & $9(25)$ & $6(16.7)$ & $6(6.7)$ \\
\hline F3 Autocontrole-Civilidade & $0-12$ & $20(55.5)$ & $8(22.2)$ & $7(19.4)$ & $1(2.8)$ \\
\hline F4 Assertividade & $0-8$ & $12(33.3)$ & $5(13.9)$ & $10(27.8)$ & $9(25)$ \\
\hline SSRS-cuidador Global de HS & $0-46$ & $20(55.6)$ & $8(22.2)$ & $4(11.1)$ & $4(11.1)$ \\
\hline F1 Responsabilidade & $0-8$ & $15(41.7)$ & $3(8.3)$ & $9(25)$ & $9(25)$ \\
\hline F2 Autocontrole & $0-10$ & $16(44.4)$ & $8(22.2)$ & $8(22.2)$ & $4(11.1)$ \\
\hline F3 Afetividade-Cooperação & $0-12$ & $17(47.2)$ & $10(27.8)$ & $3(8.3)$ & $6(16.7)$ \\
\hline F4 Desenvoltura Social & $0-8$ & $10(27.8)$ & $12(33.3)$ & $8(22.2)$ & $6(16.7)$ \\
\hline F5 Civilidade & $0-8$ & $20(55.6)$ & $2(5.6)$ & $9(25)$ & $5(13.8)$ \\
\hline
\end{tabular}

\footnotetext{
${ }^{1}$ Esses dados não foram incluídos no texto do presente trabalho.
} 
sociais e problemas de comportamento dessa amostra específica. A Tabela 1 apresenta os dados descritivos do escore global e dos escores fatoriais em frequência absoluta e relativa de crianças que apresentaram repertórios deficitário, médio inferior, médio superior e elaborado em habilidades sociais.

Como é possível ressaltar a partir da Tabela 1, na autoavaliação, metade da amostra se situou com um repertório geral de habilidades sociais $(M=24,22$; $\mathrm{MED}=25 ; D P=5,39 ; \mathrm{MIN}=11 ; \mathrm{MAX}=35)^{2}$ deficitário e mais de $80 \%$ em deficitário ou médio inferior. Da mesma forma, nos escores fatoriais pode ser observado que grande parte da amostra se situou nos repertórios deficitário e médio inferior, como nos fatores F1 - Empatia-Afetividade $(n=28 ; 77,7 \%), \mathrm{F} 2$ - Responsabilidade $(n=24 ; 66,6 \%)$, F3 - Autocontrole-Civilidade $(n=28 ; 77,7 \%)$. Somente no fator F4 - Assertividade houve uma distribuição um pouco mais equilibrada, reunindo a maior quantidade de crianças com escore indicativo de repertório elaborado $(n=9 ; 25 \%)$ e menor quantidade com escore indicativo de repertório deficitário $(n=12 ; 33,3 \%)$. A maioria da amostra apresentou, portanto, escores indicativos de repertório médio superior e elaborado $(n=19 ; 52,2 \%)$, nesse fator.

De acordo com a avaliação das crianças pelos informantes, o repertório geral de habilidades sociais da $\operatorname{amostra}(M=24,92 ; \mathrm{MED}=24 ; D P=7,71 ; \mathrm{MIN}=$ 6 ; MAX $=41$ ) também se situa, em sua maioria, como deficitário e 77,8\% como deficitário ou médio inferior. O fator F3 - Afetividade-Cooperação foi o que apresentou o maior número de crianças com repertório deficitário e médio inferior $(n=27 ; 75 \%)$. Já o fator F1 - Responsabilidade apresentou o maior número de crianças com repertório médio superior e elaborado $(n$ $=18 ; 50 \%$ ).

A análise específica de cada item mostrou, ainda, que a habilidade que teve maior ocorrência na avaliação dos cuidadores foi "Pede permissão antes de sair da instituição" ( $M=1,75 ; D P=0,55)$. A maioria das crianças faz isso com muita frequência $(n=19 ; 52,8 \%)$, enquanto isso 11 crianças apresentam tal comportamento somente algumas vezes $(30 ; 6 \%)$ e seis $(16 ; 7 \%)$ nunca fazem isso. Contrariamente, "Elogia colegas pelas suas realizações" foi a habilidade que, segundo os informantes, apresenta menor ocorrência $(M=0,58$; $D P=0,64)$. Embora boa parte das crianças emita esse comportamento algumas vezes $(n=15 ; 41,7 \%), 50 \%(n$ $=18)$ nunca o emite, e apenas $8,7 \%(n=3)$ apresenta tal comportamento com muita frequência.

A Tabela 2 apresenta os dados descritivos correspondentes aos problemas de comportamento das 36 crianças avaliadas pelos respectivos cuidadores informantes (SSRS-cuidador) em frequência absoluta e relativa de crianças que apresentaram repertório não comprometido, médio inferior, médio superior e altamente comprometido em problemas de comportamento.

Como pode ser notado na Tabela 2, a maioria das crianças da amostra $(n=21 ; 58,4 \%)$ apresentou um escore médio superior e altamente comprometido em problemas de comportamento $(M=13,28$; MED $=13,5 ; D P=6,7 ; \mathrm{MIN}=2 ; \mathrm{MAX}=26)$. Especificamente, o fator F1 - Problemas de Comportamento Externalizantes foi o que reuniu maior quantidade de crianças com escore altamente comprometido $(n=$ $16 ; 44,5 \%)$ e menor quantidade com escore não comprometido $(n=7 ; 19,4 \%)$. Contrariamente, no fator

Tabela 2.

Dados Descritivos de Problemas de Comportamento das Crianças em Relação a Padrões Nota. normativos do SSRS

\begin{tabular}{|c|c|c|c|c|c|}
\hline \multirow{3}{*}{$\begin{array}{l}\text { Problemas de } \\
\text { comportamento (PC) }\end{array}$} & \multirow{3}{*}{$\begin{array}{l}\text { Variação } \\
\text { possível do } \\
\text { escore }\end{array}$} & \multicolumn{4}{|c|}{ Repertório } \\
\hline & & $\begin{array}{c}\text { Não } \\
\text { comprometido }\end{array}$ & $\begin{array}{l}\text { Médio } \\
\text { Inferior }\end{array}$ & Médio Superior & $\begin{array}{l}\text { Altamente } \\
\text { comprometido }\end{array}$ \\
\hline & & $n(\%)$ & $n(\%)$ & $n(\%)$ & $n(\%)$ \\
\hline Escore global de PC & $0-30$ & $8(22.2)$ & $7(19.4)$ & $7(19.4)$ & $14(39)$ \\
\hline F1 PC Externalizantes & $0-20$ & $7(19.4)$ & $8(22.2)$ & $5(13.9)$ & $16(44.5)$ \\
\hline F2 PC Internalizantes & $0-10$ & $10(27.8)$ & $5(13.4)$ & $14(38.4)$ & 7 (19.4) \\
\hline
\end{tabular}

${ }^{2}(M=$ média da amostra no escore global de habilidades sociais, $\mathrm{MED}=$ mediana, $D P=$ desvio padrão, $\mathrm{MIN}=$ escore mínimo da amostra, MAX = escore máximo da amostra). 
F2 - Problemas de Comportamento Internalizantes, de acordo com os informantes, situou-se um maior grupo de crianças com escore não comprometido $(n$ $=10 ; 27,8 \%$ e menor grupo com escore altamente comprometido ( $n=7 ; 19,4 \%)$. No entanto, a maioria das crianças apresentou escores (na avaliação do cuidador) que situam o repertório como médio em termos de problemas de comportamento $(n=19$; $51,8 \%)$ nesse fator.

Em concordância com esses dados, uma análise específica de cada um dos comportamentos considerados na escala permitiu observar que, segundo a avaliação dos informantes, o problema que ocorreu com maior frequência entre as crianças foi "Discute com os outros" ( $M=1,22 ; D P=0,63)$, que faz parte dos problemas externalizantes. De acordo com os dados, 33,3\% das crianças $(n=12)$ apresentaram esse comportamento muito frequentemente, $55,6 \%$ algumas vezes $(n=20)$ e somente $11,1 \%(n=4)$ nunca o apresentaram. Por outro lado, o problema de comportamento relatado pelos informantes com menor frequência foi "Tem baixa autoestima" $(M=0,56 ; D P$ $=0,69)$, do F2 - Internalizante. A maior parte dos estudantes $(n=20 ; 55,6 \%)$ nunca apresentou esse comportamento, 33,3\% $(n=12)$ o apresentaram algumas vezes e apenas $11,1 \%(n=4)$ apresentaram esse comportamento com muita frequência.

Tabela 3.

Correlações entre Habilidades Sociais - Fatores e Escore Geral, na Autoavaliação - e Problemas e Comportamento

\begin{tabular}{lcc}
\hline Variável & HS-cri & PC \\
\hline F1 - Empatia-Afetividade & $0,609^{* * *}$ & $-0,107$ \\
F2 - Responsabilidade & $0,728^{* * *}$ & $-0,248$ \\
F3 - Autocontrole-Civilidade & $0,718^{* * *}$ & $-0,443^{*}$ \\
F4 - Assertividade & $0,537 * * *$ & $-0,298$ \\
HS-cri & 1 & $-0,425^{*}$ \\
PC & $-0,425^{*}$ & 1 \\
\hline
\end{tabular}

Nota. *Correlação significativa (valor $-p<0,05$ ), ***Correlação altamente significativa (valor $-p<0,01$ ). HS-cri: Repertório global de habilidades sociais da criança, autoavaliação; PC: Problemas de comportamento da criança, avaliação do cuidador.
$\mathrm{Na}$ Tabela 3, apresentam-se os coeficientes de correlação ( $r$ de Pearson) e significância estatística referentes às relações entre habilidades sociais - cada escore fatorial e escore geral, ambos na autoavaliação e problemas de comportamento.

Como pode ser visto na Tabela 3 , algumas variáveis se correlacionaram com valores que estão no intervalo $(0,4$ a 0,6 e 0,7 a 0,9$)$. Dessa maneira, são consideradas correlações moderadas e fortes, respectivamente (Dancey \& Reidy, 2013), com evidência de significância estatística. As correlações significativas são descritas a seguir.

1. Os fatores F1, F2, F3 e F5 se correlacionaram positivamente com o escore global de habilidades sociais da criança, na avaliação do cuidador. F1 - Responsabilidade e F5 - Civilidade, apresentaram uma correlação moderada, já F2 - Autocontrole e F3 - Afetividade-Cooperação, forte.

2. O escore geral de habilidades sociais da criança se correlacionou negativa e fortemente com o escore de problemas de comportamento, ambos na avaliação do cuidador.

3. Os fatores F1, F2, F3 e F5 de habilidades sociais da criança se correlacionaram negativamente com o escore global de problemas de comportamento, ambos na avaliação do cuidador. F1 - Responsabilidade e F5 - Civilidade apresentaram uma correlação moderada, já F2 - Autocontrole e F3 - Afetividade-Cooperação.

Tabela 4.

Correlações entre Habilidades Sociais - Fatores e Escore Geral, Avaliação do Cuidador - e Problemas de Comportamento

\begin{tabular}{lcc}
\hline Variável & HS-cri/cui & PC \\
\hline F1 - Responsabilidade & $0,674^{* * *}$ & $-0,516^{*}$ \\
F2 - Autocontrole & $0,844^{* * *}$ & $-0,723^{* * *}$ \\
F3 - Afetividade-Cooperação & $0,856^{* * *}$ & $-0,752^{* * *}$ \\
F4 - Desenvoltura Social & 0,032 & $-0,183$ \\
F5 - Civilidade & $0,668^{* * *}$ & $-0,678^{* * *}$ \\
HS-cri/cui & 1 & $-0,804 * * *$ \\
PC & & 1 \\
\hline
\end{tabular}

Nota. $*$ Correlação significativa (valor $-p<0,05$ ), $* * *$ Correlação altamente significativa (valor $-p<0,01$ ). HS-cri/cui: Repertório global de habilidades sociais da criança, na avaliação do cuidador; PC: Problemas de comportamento da criança, avaliação do cuidador. 
$\mathrm{Na}$ Tabela 4, são apresentados os coeficientes de correlação ( $r$ de Pearson) e significância estatística referentes às relações entre habilidades sociais - considerando escore geral e fatorial na autoavaliação - e problemas de comportamento, na avaliação do cuidador.

Como pode ser verificado na Tabela 4, algumas variáveis apresentaram correlações consideradas moderadas e fortes, de 0,4 a 0,6 e 0,7 a 0,9, respectivamente (Dancey \& Reidy, 2013), além de evidência de significância estatística. As correlações significativas são descritas a seguir.

1) Todos os fatores (F1, F2, F3, F4) se correlacionaram positivamente com o escore global de habilidades sociais da criança, na autoavaliação. F1 - Empatia-Afetividade e F4 - Assertividade, apresentaram uma correlação moderada, já F2 Responsabilidade e F3 - Autocontrole-Civilidade, forte.

2) O escore de habilidades sociais da criança, na autoavaliação, correlacionou-se negativa e moderadamente com o escore de problemas de comportamento, na avaliação do cuidador.

3) Apenas o fator F3 - Autocontrole-Civilidade se correlacionou com o escore geral de problemas de comportamento, na avaliação do cuidador, de maneira negativa e moderadamente.

\section{Discussão}

Para uma melhor compreensão, serão discutidos os dados de caracterização do repertório de habilidades sociais e problemas de comportamento da amostra de crianças institucionalizadas. Em seguida, discute-se as relações entre essas variáveis e necessidades de maior investimento no desenvolvimento socioemocional dessas crianças.

Quanto às habilidades sociais, o repertório da maioria das crianças dessa amostra na autoavaliação foi classificado como deficitário e médio inferior, tanto no escore geral como em três escores fatoriais. Pode-se dizer que esse resultado contrariou o viés da desejabilidade social, conforme descrito na literatura com relação à tendência das crianças, principalmente, com problemas de comportamento, de se autoavaliarem de forma muito positiva (Gresham, Lane, Macmillan, Bocian, \& Ward, 2000). Sobre os escores fatoriais, as crianças se autoavaliaram, no entanto, com um pouco mais de recursos nas habilidades de Assertividade (mais da metade com repertório médio superior e elaborado), em comparação com os demais fatores. Os maiores déficits ocorreram em habilidades de empatia. Esse é um dado preocupante, pois essa classe é considerada extremamente importante na infância e sua falta tem sido considerada como um dos fatores de comportamento antissocial e violento (Del Prette \& Del Prette, 2011), uma vez que inclui comportamentos de interesse e respeito pelos sentimentos e ponto de vista dos outros, assim como de expressão de sentimento positivo e solidariedade.

Conforme visto, não obstante à tendência filogenética para a empatia, o seu desenvolvimento está ligado à influência mútua do temperamento, desenvolvimento cognitivo, estilos parentais e o relacionamento entre pais e filhos (Justo et al., 2014). Esses fatores e a forma como se combinam na história da criança podem determinar se irão desenvolver ou não essas habilidades ao longo do desenvolvimento, especialmente se não contarem com a empatia do ambiente social como modelo e fator de proteção (Del Prette \& Del Prette, 2005). São variáveis que contribuem, portanto, para explicar os déficits em empatia da amostra, assim como de habilidades sociais no geral, bem como os altos escores em problemas de comportamento externalizantes, que são considerados concorrentes com as habilidades sociais.

Esses resultados confirmam, portanto, os de outros estudos que, desde o século passado, já vinham apontando os déficits de empatia em crianças negligenciadas ou maltratadas por colegas e familiares (Emery, 1989). Mesmo reconhecendo que as instituições de acolhimento não são a causa dos déficits em habilidades sociais das crianças, entende-se que cabe a elas, com o suporte legal e financeiro dos órgãos públicos, garantir-lhes um contexto protetor e favorável para amenizar, reduzir e superar os problemas causados pelas experiências familiares anteriores, tal como geralmente ocorre no caso de crianças adotadas (Palacios, Moreno, \& Román, 2013). Pode-se defender também a importância da atuação de profissionais de saúde e educação, com programas destinados a essa população específica, com fins de ampliar o repertório de habilidades sociais e, prioritariamente, os da classe de empatia, como apoio a essa política.

Os cuidadores, alvos deste estudo, avaliaram as crianças como menos habilidosas do que elas próprias se avaliaram, no escore geral de habilidades sociais, porém esse padrão não foi uniforme em todos os escores fatoriais: em alguns, os cuidadores superestimaram 
e, em outros, subestimaram o repertório de habilidades sociais das crianças em relação à autoavaliação que elas fizeram. Uma hipótese para isso pode ser a diferença nos itens específicos das versões do instrumento. No SSRS-cuidador (adaptado para este estudo) foram incluídos itens referentes, principalmente, no ambiente da instituição (ex., $\mathrm{Na}$ instituição, fala em tom de voz adequado), enquanto o SSRS-criança avalia comportamentos pertinentes ao contexto escolar (ex., Eu deixo a minha carteira limpa e arrumada) e à relação com os amigos em geral, não necessariamente do ambiente institucional (ex., Eu demonstro ou digo aos meus amigos que gosto deles).

Em uma análise mais detalhada, por item, os cuidadores avaliaram o desempenho das crianças como mais frequente no item "Pede permissão antes de sair da instituição". Esse dado pode ser explicado devido à proibição clara de sair da instituição sem comunicar previamente, sinalizando uma demanda relevante para as crianças. Interessante notar que o item equivalente em estudo com pais, "Pede permissão antes de sair de casa" também foi o mais frequente na avaliação dos pais sobre o desempenho das crianças, sugerindo a funcionalidade desse tipo de demanda para as crianças em geral. Contrariamente, "Elogia colegas pelas suas realizações" foi o item com menor ocorrência na avaliação dos cuidadores sobre as crianças, o que poderia ser explicado, em parte, por esse item compor o F3 Afetividade/Cooperação, relacionado ao F1 - Empatia, da escala de autoavaliação, que, na amostra estudada, foram os mais deficitários.

Quanto aos problemas de comportamento, avaliados pelos cuidadores, a maioria da amostra apresentou repertório médio superior e altamente comprometido no escore geral, com escores mais altos para problemas externalizantes. $\mathrm{Na}$ literatura, não há consenso sobre a prevalência de problemas externalizantes ou internalizantes, com evidências discrepantes para um ou outro (Casali-Robalinho, 2013). No presente estudo, cabe questionar se os problemas internalizantes são de fato menos frequentes ou se são menos percebidos ou considerados menos problemáticos pelos cuidadores.

Em consonância com esses dados, considerando os itens específicos, o problema de comportamento mais pontuado pelos cuidadores foi "Discute com os outros", que faz parte dos problemas externalizantes. Por outro lado, o problema de comportamento indicado com menor frequência foi "Tem baixa autoestima" do conjunto dos internalizantes. É provável que os cuidadores se preocupem mais com crianças que apresentam problemas externalizantes, mais difíceis de ignorar e que geram mais demanda de trabalho para eles. Os externalizantes são mais visíveis e trazem prejuízos, não só à criança, mas também às pessoas que estão ao redor dela, enquanto que os problemas internalizantes raramente trazem incômodo aos outros (Wielewicki, 2011). Seria importante para uma atuação dos cuidadores mais favorável ao desenvolvimento infantil, que eles reconhecessem que ambos são problemáticos: enquanto os comportamentos internalizantes privam a criança de interagir no ambiente social da instituição (isolamento), os externalizantes podem provocar rejeição social dos pares e adultos significativos (Del Prette \& Del Prette, 2005).

Nessa direção, os resultados do presente estudo sugerem que as crianças institucionalizadas apresentam déficits no desenvolvimento socioemocional, em termos de repertório deficitário em habilidades sociais e altamente comprometido em problemas de comportamento. Com relação aos problemas de comportamento, a revisão de Wielewicki (2011), com crianças na faixa etária de 7 a 12 anos, mostrou que elas eram mais frequentemente encaminhadas para atendimento psicológico, em detrimento das crianças de 0 a 6 e de 13 a 14 anos, respectivamente. Isso pode sugerir uma relação entre faixa etária e problemas comportamentais, que explicaria a não diferenciação dos escores de problemas de comportamento da amostra, já que estão todos dentro dessa faixa.

No que se refere às relações entre variáveis, o escore global de habilidades sociais das crianças se correlacionou negativamente com o escore global de problemas de comportamento, tanto na autoavaliação quanto na avaliação do cuidador, sendo moderada e forte, respectivamente. Os resultados são coerentes com a literatura, que aponta uma relação bem estabelecida entre um repertório mais elaborado de habilidades sociais e menor ocorrência de problemas de comportamento (Bolsoni-Silva, Loureiro, \& Marturano, 2011; Gresham, 2013). Na avaliação do cuidador, as crianças estariam no ápice da condição desfavorável em termos de fatores de risco (problemas de comportamento) e de proteção (habilidades sociais) para um desenvolvimento socioemocional saudável.

Os escores fatoriais de habilidades sociais da criança se correlacionaram positivamente, moderada e fortemente, com o seu respectivo escore geral, tanto na autoavaliação quanto na avaliação do cuidador - com apenas uma exceção para o fator F4 - Desenvoltura social. Esses resultados são esperados, de acordo com 
a literatura (Gresham \& Elliot, 2016). No entanto, considera-se relevante ressaltar os maiores índices de correlação que poderiam ser priorizados e enfatizados em programas de treinamento de habilidades sociais, nesse contexto específico.

O escore de habilidades sociais de Autocontrole-Civilidade (F3) na autoavaliação da criança, foi o único que se correlacionou com o escore geral de problemas de comportamento, um resultado coerente com os de outros estudos (Bolsoni-Silva \& Del Prette, 2003; Brasil, 2014; Casali-Robalinho, 2013). Esse dado sugere um objetivo altamente relevante para esses programas. Comportamentos como responder adequadamente a provocações, recusar educadamente pedidos abusivos ou falar em tom de voz apropriado demonstram controle sobre suas próprias emoções parecem, conforme os dados da presente pesquisa, concorrerem com problemas comportamentais nas crianças avaliados pelos cuidadores.

$\mathrm{Na}$ avaliação dos cuidadores, o único fator que não se correlacionou com os problemas de comportamento foi o que reúne as habilidades de Desenvoltura Social (F4), composto por comportamentos como juntar-se a grupos de atividade sem ser mandado, apresentar-se a novas pessoas e iniciar conversações. Uma hipótese é que as habilidades desse fator foram deficitárias para uma minoria de crianças e associadas a problemas internalizantes, como tristeza, retraimento, timidez, insegurança, medos e inibição excessiva, que também foram menos frequentes na amostra de crianças. As demais classes de habilidades sociais da criança, na avaliação do cuidador, reunidas nos fatores F1 - Responsabilidade, F2 - Autocontrole, F3 - Afetividade- Cooperação e F5 - Civilidade, se relacionaram negativamente com os problemas de comportamento, na medida em que de fato competem e concorrem com eles (Del Prette \& Del Prette, 2005).

No presente trabalho, não se utilizou a escala do SSRS de importância das habilidades sociais. No entanto, Brasil (2014) encontrou que as habilidades de cooperação, autocontrole e civilidade foram as mais valorizadas pelos pais. A amostra de crianças institucionalizadas apresentou, justamente nessas três classes de habilidades sociais, os maiores déficits, conforme a avaliação do cuidador, assim como os maiores coeficientes de correlação com os problemas de comportamento, sugerindo objetivos para programas de intervenção. Mesmo considerando que são dados correlacionais, os resultados obtidos, portanto, sugerem a importância de investir, primordialmente, nas classes de habilidades sociais de cooperação, autocontrole e civilidade de crianças institucionalizadas para a prevenção e redução de problemas de comportamento.

Os dados do presente estudo poderiam, portanto, indicar ou sugerir direções para intervenções relevantes em ambientes institucionais de acolhimento e, na medida em que devidamente testados e aprovados, serem incorporados em políticas públicas para esse setor. Pelo menos duas direções poderiam ser relevantes a partir deste estudo. A primeira é certamente um maior investimento público nas condições físicas das instituições de acolhimento para que se tornem ambientes mais favoráveis ao desenvolvimento das crianças. A segunda, possivelmente ainda mais importante, é a capacitação dos profissionais que trabalham em serviços de assistência social, de modo a exercerem uma influência positiva mais direta na promoção do desenvolvimento dessas crianças, inclusive daquelas com repertório de comportamentos que dificulta os esforços de interação. Em estudo conduzido na Rússia (McCall et al., 2013), os autores demonstraram a eficácia e a sustentabilidade dos resultados de um programa com seis anos de follow- up em que compararam uma alternativa que incluiu treinamento dos cuidadores associada a mudanças estruturais, a outra apenas com o treinamento e ambas a resultados obtidos em uma instituição controle sem intervenção. Os resultados indicaram redução dos escores no Battelle Developmental Inventory Scores for Children, que avalia atrasos desenvolvimentais e dificuldades na aprendizagem, em crianças.

Nessa segunda direção, estudos promissores em nosso país, com a utilização de referencial teórico-prático das habilidades sociais e de recursos e procedimentos específicos, poderiam ser utilizados diretamente com as crianças por profissionais especializados estruturando condições efetivas de ensino-aprendizagem de habilidades sociais ou capacitando os cuidadores para isso (Del Prette \& Del Prette, 2005). Em ambos os casos, o foco seria o investimento em um desenvolvimento satisfatório, que poderia reduzir o risco de uma trajetória problemática para a criança e para seu entorno.

Considerando o conhecimento sobre intervenções, produzido em outros países, pode-se destacar a revisão de literatura sobre os efeitos do cuidado institucional, realizada por Dozier, Zeanah, Wallin e Shauffer (2012). Esses autores focalizaram um modelo de cuidado implementado na Romênia, o Bucharest Early Intervention Project (BEIP), testado a partir de ensaio clínico randomizado. As crianças sob cuidados institucionais foram avaliadas e, em seguida, 
aleatoriamente designadas para instituições de maior ou menor qualidade no cuidado. Os resultados permitiram aos autores recomendar cuidados específicos no atendimento às crianças e mudanças nas políticas públicas, tais como reconhecer impedimentos à transição dos cuidados institucionais (muitas vezes sobrevivem de donativos e empregam muitas pessoas), desenvolver um sistema de apoio às famílias e comunidades, garantir suporte para pais biológicos e fortalecer a rede de parentesco extensa (tios, avós).

A busca de evidências para selecionar intervenções bem-sucedidas como as citadas anteriormente constitui uma direção importante de pesquisa para encaminhar a solução ou redução dos problemas de crianças em situação de acolhimento institucional. Não se trata, porém, de importar acriticamente programas de outros países, que requerem adaptações culturais, mas de investir, também, em programas criados e delineados para nosso contexto cultural.

\section{Considerações Finais}

Os resultados do presente estudo trazem evidências adicionais sobre as condições desfavoráveis ao desenvolvimento socioemocional das crianças em situação de acolhimento. Não obstante, cabe reconhecer as limitações do estudo, no que diz respeito à generalização de resultados, já que os dados foram coletados por amostragem não probabilística, o que limita sua generalização. Pesquisas futuras poderiam utilizar o referencial teórico das Habilidades Sociais Educativas (Del Prette, \& Del Prette, 2013) para formular e testar novos programas de intervenção e suas possibilidades de disseminação em políticas públicas nessa área.

\section{Referências}

Bakermans-Kranenburg, M. J., Van IJzendoorn, M. H., \& Juffer, F. (2008). Earlier is better: A meta-analysis of 70 years of intervention improving cognitive development in institutionalized children. Monographs of the Society for Research of Child Development, 73(3), 279-293. doi: 10.1111/j.1540-5834.2008.00498.x.

Bolsoni-Silva, A. T., \& Del Prette, A. (2003). Problemas de comportamento: Um panorama da área. Revista Brasileira de Psicologia e Medicina Comportamental, 5(2), 91-103. Recuperado de http://pepsic. bvsalud.org/scielo.php?script $=$ sci_arttext\&pi$\mathrm{d}=$ S1517-55452003000200002
Bolsoni-Silva, A. T., Loureiro, S. R., \& Marturano, E. M. (2011). Problemas de comportamento e habilidades sociais infantis: Modalidades de relato. Psico, 42(3), 354-361. Recuperado de http://revistaseletronicas.pucrs.br/ojs/index.php/revistapsico/ article/view/5813

Brasil, S. E. R. (2014). Habilidades sociais parentais e infantis, problemas de comportamento em pré-escolares: Avaliação de pais e professores (Dissertação de mestrado). Universidade Federal de São Carlos, São Carlos.

Casali-Robalinho, I. G. (2013). Relacõoes entre automonitoria, problemas de comportamento e habilidades sociais na infância (Dissertação de mestrado). Universidade Federal de São Carlos, São Carlos.

Cardeira, C., Almeida, A., Martins, C., Bento, M., \& Cabeleira, F. (2011). Perfil de desenvolvimento global de crianças institucionalizadas e não institucionalizadas. Internacional Journal of Developmental and Educational Psychology, 1(1), 379-388. Recuperado de http:/ / infad.eu/RevistaINFAD/2011/n1/volumen1/INFAD_010123_379-388.pdf

Cecconello, A. M., \& Koller, S. H. (2000). Competência social e empatia: Um estudo sobre resiliência com crianças em situação de pobreza. Estudos de Psicologia, 5(1), 71-93. doi: 10.1590/ S1413-294X2000000100005

Chaves, C. M. P., Lima, F. E. T., Mendonça, L. B. A., Custódio, I. L., \& Matias, E. O. (2013). Avaliação do crescimento e desenvolvimento de crianças institucionalizadas. Revista Brasileira de Enfermagem, 66(5), 668-674. doi: 10.1590/S0034-71672013000500005

Dancey, C. P., \& Reidy, J. (2013). Estatística sem matemática para psicologia. Porto Alegre: Artmed.

Del Prette, Z. A. P., \& Del Prette, A. (2005). Psicologia das habilidades sociais na infância: Teoria e Prática. Petrópolis: Vozes.

Del Prette, Z. A. P., \& Del Prette, A. (2011). Psicologia das Habilidades Sociais: Terapia, educação e trabalho. Petrópolis: Vozes.

Del Prette, Z. A. P., \& Del Prette, A. (2013). Psicologia das Relações Interpessoais: Vivências para o trabalho em grupo. Petrópolis: Vozes.

Del Prette, A., \& Del Prette, Z. A. P. (2017). Habilidades sociais: Intervenções efetivas em grupo. São Paulo: Pearson. 
Del Prette, Z. A. P., Prette, A. D., De Oliveira, L. A., Gresham, F. M., \& Vance, M. J. (2012). Role of social performance in predicting learning problems: Prediction of risk using logistic regression analysis. School Psychology International, 33(6), 615-630. doi:10.1177/0020715211430373

Dozier, M., Zeanah, C. H., Wallin, A. R., \& Shauffer, C. (2012). Institutional care for young children: Review of literature and policy implications. Social Issues and Policy Review, 6(1), p. 1-25. doi: 10.1111/j.1751-2409.2011.01033.x

Emery, R. E. (1989) Family violence. Special Issue: Children and their development: Knowledge base, research agenda, and social policy application. American Psychologist, 44(2), 321-328. doi: 10.1037/0003-066X.44.2.321

Freitas, L. C., \& Del Prette, Z.A.P. (2015). Social Skills Rating System - Brazilian version: New exploratory and confirmatory factorial analyses. Avances en Psicología Latinoamericana /Bogotá (Colombia), 33(1), 135-156.

Gaspar, A. (2014). Neurobiologia e psicologia da empatia. Pontos de partida para a investigação e intervenção da promoção da empatia. Em P. Henenberg and A. C Caldas (Orgs.), Cérebro: O que a ciência nos diz. Povos e Culturas, 18, 159-174.

Gresham, F. M. (2013). Análise do Comportamento aplicada às habilidades sociais. In Almir Del Prette; Zilda A.P. Del Prette (Organizadores), Psicologia das habilidades so-ciais: diversidade teórica e suas implicações - 3 ed. Petrópolis, RJ: Vozes.

Gresham, F. M., Lane, K. L., Macmillan, D. L., Bocian, K. M., \& Ward, S. L. (2000). Effects of positive and negative illusory biases: Comparison across social and academic self-concept domains. Journal of School Psychology, 38, 151-175. doi: 10.1016/ S0022-4405(99)00042-4

Gresham, F. M., \& Elliott, S. N. (2016). SSRS - Inventário de Habilidades Sociais, Problemas de Comportamento e Competência Acadêmica para Crianças [Adaptação Brasileira de Z. A. P. Del Prette, L. C. de Freitas, M. B. Bandeira, \& A. Del Prette]. São Paulo: Pearson.

Hodel, A. S., Hunt, R. H., Cowell, R. A., Van Den Heuvel, S. E., Gunnar, M. R., \& Thomas, K. M. (2015). Duration of early adversity and structural brain development in post-institutionalized adolescents.
NeuroImage, 105, p. 112-119. doi: doi: 0.1016/j. neuroimage.2014.10.020

Justo, A. R., Carvalho, J. C. N., \& Kristensen, C. H. (2014). Desenvolvimento da empatia em crianças: A influência dos estilos parentais. Psicologia, Saúde \& Doenças, 15(2), 510-523. doi: 10.15309/14psd150214

Lei no .8069 , de 13 de julho de 1990 (1990). O estatuto da crianç e do adolescente (ECA). Brasília, DF.

Levin, A. R., Fox, N. A., Zeanah Jr, C. H., \& Nelson, C. A. (2015). Social communication difficulties and autism in previously institutionalized children. Journal of the American Academy of Child \& Adolescent Psychiatry, 54(2), p. 108-115. doi: 10.1016/j. jaac.2014.11.011

Lionettia, F., Pastore, M., P., \& Barone, L. (2015). Attachment in institutionalized children: A review and meta-analysis. Child Abuse \& Neglect, 42, p. 135-145. doi: 10.1016/j.chiabu.2015.02.013

Lima, A. P.,Lima, A. O. (2012). Perfil do desenvolvimento neuropsicomotor e aspectos familiares de crianças institucionalizadas na cidade do Recife. Revista CES Psicologia, 5(1), 11-25. Recuperado de http:// www.redalyc.org/pdf/4235/423539529003.pdf

McCall, R. B., Groark, C. J., Fish, L., Muhamedrahimov, R. J., Palmov, O. I., \& Nikiforova, N. V. (2013). Maintaining a social-emotional intervention and its benefits for institutionalized children. Child Development, 84(5), p. 1734-1749. doi: 10.1111/cdev.12098

Palacios J., Moreno C., \& Román M. (2013). Social competence in internationally adopted and institutionalized children. Early ChildhoodResearch Quarterly, 28, 357-365. doi: 10.1016/j.ecresq.2012.08.003

Troller-Renfree, S., McDermott, J. M., Nelson, C. A., Zeanah, C. H., \& Fox, N. A. (2015). The effects of early foster care intervention on attention biases in previously institutionalized children in Romania. Developmental Science, 18(5), p. 713-722. doi: $10.1111 /$ desc. 12261

Wielewicki, A. (2011). Problemas de comportamento infantil: Importância e limitações de estudos de caracterização em clínicas-escola brasileiras. Temas em Psicologia, 19(2), 379-389. Recuperado de http:// pepsic.bvsalud.org/pdf/tp/v19n2/v19n2a03.pdf

Recebido em: 11/12/2017 Reformulado em: 12/02/2019 
Sobre as autoras:

Lívia Lira de Lima Guerra é mestre em Psicologia pelo Programa de Pós-Graduação em Psicologia (PPGPsi) da Universidade Federal de São Carlos - UFSCar (2017). Atualmente é doutoranda com ênfase em Comportamento Social e Processos Cognitivos, pelo mesmo programa e atua na área de Psicologia do Desenvolvimento Adulto, com temas em coparentalidade, habilidades sociais e parentalidade.

ORCID: https://orcid.org/0000-0002-2534-4462

E-mail:psicologa@livialira.com.br

Zilda Aparecida Pereira Del Prette é doutora em Psicologia, com pós-doutorado em Habilidades Sociais, professora titular sênior vinculada aos departamentos de graduação e pós-graduação de Psicologia e Educação Especial da Universidade Federal de São Carlos - UFSCar e bolsista de produtividade em Pesquisa do CNPq nível 1A.

ORCID: https://orcid.org/0000-0002-0130-2911

E-mail: zdprette@ufscar.br

Contato com as autoras:

Universidade Federal de São Carlos, Departamento de Psicologia, Grupo Relações Interpessoais e Habilidades Sociais (RIHS)

Laboratório de Interação Social (LIS), Rodovia Washington Luís, Km 235

São Carlos-SP, Brasil

CEP: 13565-905 